Originalien

Gefässchirurgie $2020 \cdot 25: 433-445$ https://doi.org/10.1007/s00772-020-00677-6 Online publiziert: 7. August 2020

(c) Der/die Autor(en) 2020

\section{A. Kühnl · E. Knipfer · T. Lang • B. Bohmann · M. Trenner · H.-H. Eckstein}

Klinik und Poliklinik für Vaskuläre und Endovaskuläre Chirurgie, Klinikum rechts der Isar, Technische Universität München, München, Deutschland

\title{
Krankenhausinzidenz, stationäre Versorgung und Outcome der peripheren arteriellen Verschlusskrankheit und arteriellen Thrombose/Embolie in Deutschland von 2005 bis 2018
}

\section{Zusatzmaterial online}

Die Online-Version dieses Beitrags (https:// doi.org/10.1007/s00772-020-00677-6) enthält die ergänzenden Tab. 5 und 6 sowie die Abb. 7. Beitrag und Zusatzmaterial stehen Ihnen auf www.springermedizin. de zur Verfügung. Bitte geben Sie dort den Beitragstitel in die Suche ein, das Zusatzmaterial finden Sie beim Beitrag unter „Ergänzende Inhalte".

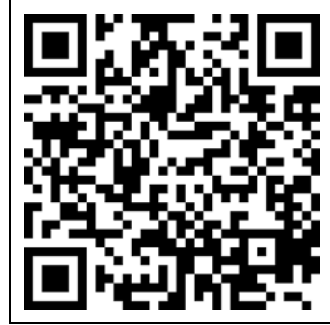

\section{Einleitung}

Die Prävalenz der peripheren arteriellen Verschlusskrankheit (pAVK) liegt in den industrialisierten Ländern bei Personen über 60 Jahren bei ca. 10-20\%, wobei ein Großteil der Patienten asymptomatisch ist [1-3]. In einer systematischen Literaturanalyse wird die Zahl der weltweit an einer pAVK Erkrankten auf ca. 200 Mio. geschätzt [3, 4]. Im Allgemeinen sind Männer häufiger betroffen als Frauen und ältere Menschen häufiger als jüngere. Eine auf die ethnische Herkunft fokussierte Analyse aus den USA zeig- te, dass die Prävalenz der pAVK besonders in der Gruppe der über 40-jährigen männlichen „African Americans“ (8,8\% im Durchschnitt, 24-59\% bei >70-Jährigen) und der „American Indians“ (6,1\% im Durchschnitt, 12-29\% bei >70-Jährigen) deutlich höher war als bei USBürgern asiatischer, spanischer oder europäischer Herkunft [3, 5].

Aus globaler Sicht ist die Prävalenz der pAVK in Ländern mit hohem und mittlerem Einkommen niedriger als in ärmeren Ländern [4]. Ebenso zeigte sich ein Zusammenhang zwischen niedrigem sozioökonomischem Status und einer hohen Prävalenz der pAVK [4]. Zum Teil war dies durch die Raucherprävalenz zu erklären, aber nicht durch andere Risikofaktoren [4]. Paradoxerweise stellt in Ländern mit hohem Einkommen gerade ein hoher soziökonomischer Status einen Risikofaktor dar, vermutlich bedingt durch die westlichen Lebens- und Ernährungsgewohnheiten [4]. Eine ausführliche Darstellung und Diskussion dieser und weiterer Risikofaktoren findet sich bei Fowkes et al. [4]. Die pAVK weist aufgrund der vergleichbaren Pathophysiologie eine hohe Co-Inzidenz mit anderen kardiovaskulären Erkrankungen, wie z.B. der koronaren Herzkrankheit (KHK) oder der Carotisstenose auf und ist selbst ein Risikofaktor für eine erhöhte Mortalität. Im REACH-Register konnte z. B. gezeigt werden, dass $39 \%$ aller pAVK-Patienten eine begleitende KHK und $10 \%$ eine Carotisstenose hatten [3, 4]. Die Sterblichkeit (jeglicher Ursache) war in einer Metaanalyse bei symptomatischer pAVK im Vergleich zu Patienten ohne pAVK signifikant erhöht (Mortality Ratio $=1,98$ ) [4]. Auch bereits bei asymptomatischer pAVK zeigte sich eine signifikant erhöhte Sterblichkeit (Mortality Ratio=1,53). In einer auf Krankenkassendaten basierenden Arbeit von Reinecke et al. konnte mit einem multivariablen Cox-Regressionsmodell gezeigt werden, dass die Stadien der pAVK signifikante Prädiktoren für Tod, Amputation, Myokardinfarkt und Schlaganfall sind [6]. Zu den Risikofaktoren zählen Rauchen, Diabetes mellitus, arterieller Hypertonus, Hypercholesterinämie sowie Adipositas und das Lebensalter. Aufgrund der demographischen Entwicklung ist damit auch in Deutschland mit einer weiteren Zunahme der pAVK zu rechnen.

Die stationäre Diagnostik und Behandlung der pAVK und peripheren Thrombose und Embolie erfolgt in Deutschland überwiegend in eigenständigen Fachabteilungen für Gefäßchirurgie oder ungeteilten Abteilungen für Chirurgie [1, 7]. Um die Folgen der pAVK und der mit ihr assoziierten Erkrankungen (kritische Extremitätenischämie, Amputationen, Herzinfarkt, 
Schlaganfall etc.) zu verhindern, ist eine frühe Diagnostik und stadiengerechte Therapie notwendig. Leitlinien zur Diagnostik und Therapie wurden kürzlich auf europäischer Ebene konsentiert und publiziert [8]. Daneben spielen im ambulanten wie im stationären Bereich ein hohes Problembewusstsein und Qualifikation des medizinischen Personals, wie auch eine flächendeckend gute Versorgungsstruktur eine wichtige Rolle. $\mathrm{Zu}$ deren Planung und Ressourcensteuerung sind aktuelle Daten zu Inzidenz, Versorgungsbedarf und Outcome unerlässlich.

In der vorliegenden Studie, die methodisch analog zu früheren Publikationen unserer Arbeitsgruppe aufgebaut ist $[1,7,9]$, wurde daher die zeitliche Entwicklung der Krankenhausinzidenz sowie der durchgeführten operativen und endovaskulären Prozeduren von 2005 bis 2018 analysiert. Des Weiteren wird im Hinblick auf die Krankenhausmortalität, die behandelnden Fachabteilungen sowie die altersgruppen- und bundeslandspezifische Inzidenz der Status quo der Berichtsjahrgänge 2017 bzw. 2018 deskriptiv dargestellt.

\section{Methoden}

Die angewandten Methoden wurden bereits mehrfach in früheren Studien unserer Arbeitsgruppe verwendet und im Detail publiziert $[1,7,9]$.

\section{Datenquellen}

Zur Analyse der Krankenhausinzidenz wurde die fallpauschalenbezogene Krankenhausstatistik (DRG-Statistik) des statistischen Bundesamtes der Jahre 2005 bis 2018 verwendet, die sog. ICD-10GM 5-Steller. Diese umfassen alle vollstationären Patientinnen und Patienten in deutschen Krankenhäusern, einschließlich Sterbe- und Stundenfälle, die nach dem DRG-Vergütungssystem abrechnen und dem Anwendungsbereich des $₫ 1$ KHEntgG unterliegen. Zur Optimierung und Weiterentwicklung der bisherigen amtlichen Krankenhausstatistik wird über das Institut für das Entgeltsystem im Krankenhaus (InEK) ein ausgewähltes und gesetzlich genau definiertes
Merkmalsspektrum dieser umfangreichen Struktur- und Leistungsdaten an das Statistische Bundesamt übermittelt [10]. Auf dieser Basis stehen Informationen über die fallpauschalenbezogene Krankenhausstatistik (DRG-Statistik) zur Verfügung [10]. Es handelt sich somit um eine Vollerhebung, nicht um eine Stichprobe. Aufgrund der Vollständigkeit der Datensätze mussten keine Verfahren zur Imputation von fehlenden Daten angewandt werden. Der Beobachtungszeitraum bezog sich immer auf einen administrativen DRGFall, von der Aufnahme des Patienten bis zur Entlassung. Das Problem eines "loss to follow-up“ bestand nicht. Die Erhebung bezieht sich ausschließlich auf Fälle mit den Krankenhaushauptdiagnosen für pAVK oder arterielle Thrombose und Embolie, die in Tab. 5 aufgelistet sind. Zur Analyse der Häufigkeit von Eingriffen wurde ebenfalls die DRGStatistik von 2005 bis 2018 verwendet, jedoch unter Bezug auf die Auswertung der „Operationen und Prozeduren bis zum kodierbaren Endpunkt", die sog. OPS 6-Steller. Die ICD-10-GM 5-Steller und OPS 6-Steller lagen den Autoren dieser Studie vollständig vor und wurden wie vom Statistischen Bundesamt geliefert ausgewertet. Ein zusätzliches Data Clearing fand nicht statt. Ein Zugriff auf die Mikrodaten war den Autoren nicht möglich, wohingegen die aggregierten Daten kostenpflichtig aber frei verfügbar sind. Die verwendeten Codes sind in Tab. 6 aufgelistet. Zur Analyse der Krankenhausmortalität, der versorgenden Fachabteilung, der Belegungstage sowie der bundeslandspezifischen Krankenhausinzidenz wurde der aktuellste verfügbare Berichtsjahrgang 2017 der „Tiefgegliederten Diagnosedaten der Krankenhauspatientinnen und -patienten" verwendet, da diese Analysen durch die DRG-Statistik nicht abgebildet werden konnten. Die Fachabteilungscodierung wurde dem Schlüsselverzeichnis des Statistisches Bundesamtes für die Fachserie 12, Reihe 6.2.1 entnommen. Leider sind aus Datenschutzgründen die Angaben auf Ebene der ICD 3-Steller aggregiert, sodass lediglich zwischen Atherosklerose (I70) und arterieller Embolie und Thrombose (I74) unterschie- den werden konnte. Für I70 bedeutet dies, dass auch die Atherosklerose der Aorta (I70.1), der Nierenarterie (I70.2), sonstiger Arterien (I70.8) und die generalisierte und nicht näher bezeichnete Atherosklerose (I70.9) in die Berechnung der diagnoseassoziierten Letalität eingeflossen ist. Diese Unterdiagnosen umfassen allerdings nur etwa $2 \%$ der gesamten Gruppe I70 [9]. Eine Verlinkung mit anderen Datenbanken fand nicht statt.

\section{Gruppeneinteilung und Codierung}

Alle Patienten bzw. Krankenhausfälle wurden nach ihrem Geschlecht und Alter in jeweils 7 Gruppen aufgeteilt: $<40,40-49,50-59,60-69,70-79,80-89$ und $\geq 90$. Diese Gruppierung wurde analog zu Vorpublikationen gewählt, um Vergleichbarkeit herzustellen. Die Krankenhausinzidenz (Krankenhaushäufigkeit) wurde - im Rahmen dieser Studie und bezogen auf 100.000 Einwohner - als Anzahl administrativer Fälle (DRG-Fälle) mit einer der in Tab. 5 aufgelisteten Hauptdiagnosen bezogen auf das jeweilige Berichtsjahr definiert. Nebendiagnosen standen im genutzten Datenmaterial nicht zur Verfügung. Die Einteilung der klinischen Stadien orientierte sich an den administrativen ICD10-Codes für pAVK und arterielle Embolie und Thrombose (siehe Tab. 5). Die arterielle Verschlusskrankheit der Arme wurde bewusst ausgeschlossen, da diese nicht Fokus des vorliegenden Papers war. Die Einteilung der therapeutischen Prozeduren erfolgte nach dem Operationen- und Prozedurenschlüssel (OPS) des DIMDI (www.dimdi.de) für offen-chirurgische und endovaskuläre Prozeduren, Prozeduren bei arterieller Embolie sowie für Minor- und Majoramputationen (siehe Tab. 6). Wie schon bei einer früheren Publikation [9] wurde die Diagnosegruppe I73 erneut nicht eingeschlossen, da diese Gruppe die „sonstigen peripheren Gefäßkrankheiten" abbildet, die in erster Linie funktionelle und entzündliche Gefäßerkrankungen beinhaltet (z. B. Thrombangiitis obliterans, RaynaudSyndrom etc.). Außerdem wurden die Codes für das diabetische Fußsyndrom (DFS, E10-14) nicht eingeschlossen, da 
Gefässchirurgie 2020 $25: 433-445$ https://doi.org/10.1007/s00772-020-00677-6

(c) Der/die Autor(en) 2020

A. Kühnl · E. Knipfer · T. Lang · B. Bohmann · M. Trenner · H.-H. Eckstein

Krankenhausinzidenz, stationäre Versorgung und Outcome der peripheren arteriellen
Verschlusskrankheit und arteriellen Thrombose/Embolie in Deutschland von 2005 bis 2018

\section{Zusammenfassung}

Hintergrund. Akute und chronische Durchblutungsstörungen der unteren Extremitäten sind häufig und treten mit zunehmendem Lebensalter vermehrt auf. Ziel dieser Studie ist die Erfassung der Krankenhausinzidenz peripherer Durchblutungsstörungen in Deutschland sowie der Behandlungsverfahren und der behandelnden Fachabteilungen. Methoden. Zur Analyse wurde die DRGStatistik des gesamten Bundesgebiets der Jahre 2005 bis 2018 verwendet. Zur Analyse der Krankenhausmortalität, der versorgenden Fachabteilung, der Belegungstage sowie der bundeslandspezifischen Krankenhausinzidenz wurde der aktuellste verfügbare Berichtsjahrgang 2017 der „Tiefgegliederten Diagnosedaten“ analysiert. Die Einteilung der klinischen Stadien orientierte sich an den administrativen ICD-10 Codes für die pAVKStadien I-IV.

Ergebnisse. Die altersstandardisierte Krankenhausinzidenz der PAVK I-IV betrug zuletzt 231 pro 100.000 Einwohner (EW), die der arteriellen Embolie/Thrombose 23 pro 100.000 EW. Die absolute Anzahl an offenen und endovaskulären Prozeduren sowie Prozeduren bei arterieller Embolie/Thrombose und Amputation stieg stetig von 2005 (ca. 260.000) bis 2018 (ca. 620.000) an. Offenchirurgische Eingriffe bei pAVK stiegen um $35 \%$, bei arterieller Embolie/Thrombose um $56 \%$ und endovaskuläre Verfahren um $141 \%$. Majoramputationen nahmen um $30 \%$ von 25.902 auf $17.237 \mathrm{ab}$, die der Minoramputationen von 37.102 auf 46.193 um $+21 \%$ zu. Die Krankenhausmortalität der pAVK I-IV betrug in 2017 im Mittel für
Männer 2,3 \% und für Frauen 2,8 \%, bei der arteriellen Embolie/Thrombose waren dies 6,8 und $12,0 \%$.

Schlussfolgerungen. Die Krankenhausinzidenz von Männern ist höher als die von Frauen; eine Altersabhängigkeit ist bei beiden Geschlechtern vorhanden. Deutliche Unterschiede in der Krankenhausinzidenz zeigen sich zwischen den Bundesländern. Die Anzahl an endovaskulären Prozeduren zur Behandlung einer pAVK oder arteriellen Embolie nehmen weiterhin stetig zu, während die Rate an Majoramputationen abnimmt.

Schlüsselwörter

Periphere arterielle Verschlusskrankheit . Krankenhausinzidenz - Revaskularisation . Amputation · Krankenhausmortalität

\section{Hospital incidence, in-patient care and outcome of peripheral arterial occlusive disease and arterial thrombosis/embolism in Germany, 2005-2018}

\section{Abstract}

Background. The incidence of acute and chronic peripheral arterial occlusive diseases (PAOD) of the lower limbs is high and increase even further with age. Aim of this study was to analyse the hospital incidence, type of treatment and the treating departments of peripheral occlusive disease in Germany. Methods. For the analysis, the German Diagnosis-Related Group statistics from 2005 to 2018 were used. To analyse in-hospital mortality, the treating department, the length of stay in hospital, and the federal statespecific hospital incidence, the "Detailed diagnosis-related statistics of hospital patients" were used. Clinical classification was according to the ICD-10 Codes for peripheral arterial occlusive disease (PAOD) stages I-IV.
Results. Age-standardised hospital incidence was 231 per 100,000 inhabitants for PAOD IIV, and 23 per 100,000 for arterial embolism and thrombosis. The number of open-surgery and endovascular procedures for PAOD and for arterial embolism and thrombosis increased from 260,000 in 2005 to 620,000 in 2018. Open-surgery procedures for PAOD increased by $35 \%$, for arterial embolisation and thrombosis by $56 \%$, and for endovascular procedures by $141 \%$. Major amputations decreased by $30 \%$ (from 25,902 to 17,237 ), and minor amputations increased by $21 \%$ (from 37,102 to 46,193). In 2017, in-hospital mortality of PAOD I-IV was $2.3 \%$ for men and $2.8 \%$ for women; for arterial embolisation and thrombosis, this was 6.8 and $12.0 \%$ respectively.

Conclusions. The hospital incidence is higher in men than in women, and is related to age in both sexes. Significant regional differences in hospital incidence can be seen between federal states. The number of endovascular procedures for the treatment of PAOD and arterial embolism is increasing steadily, whereas the rate of major amputations is decreasing.

Keywords Peripheral artrerial occlusive disease $\cdot$ Hospital incidence - Revascularization - Amputation . Hospital mortality 

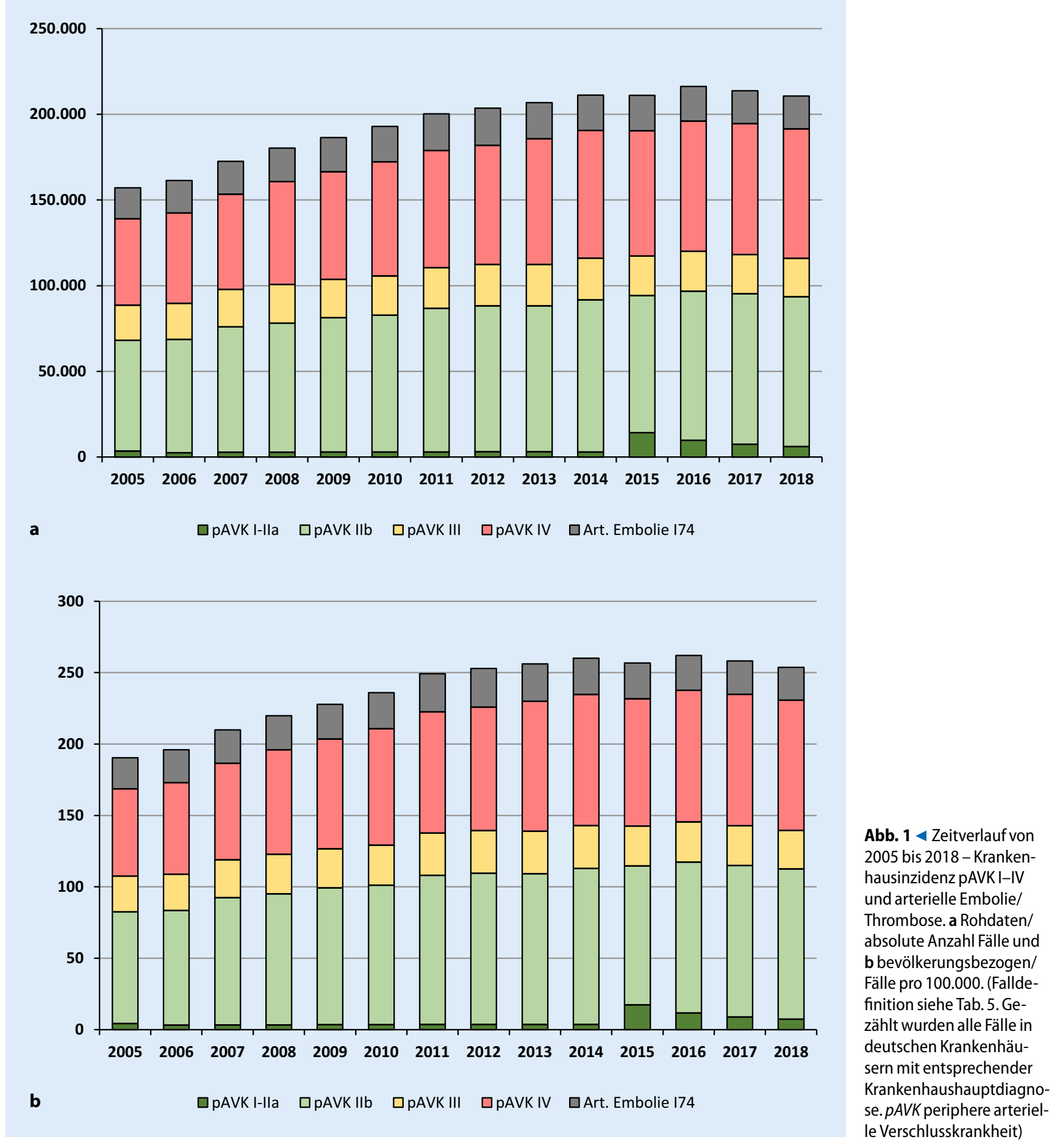

bei diesen Hauptdiagnosen der Anteil von diabetischen Patienten mit einer zusätzlichen Makroangiopathie nicht differenziert werden kann.

\section{Statistik}

Bei der vorliegenden Arbeit handelt es sich um eine deskriptive Arbeit. Um die Vergleichbarkeit der Daten über die Jahre hinweg unabhängig von demographischen Veränderungen darstellen zu können, erfolgte eine direkte Altersstandardisierung unter Verwendung der „Europastandardbevölkerung 2013“. Zur Quantifizierung des zeitlichen Trends erfolgte ein einfacher Vergleich der arithmetischen Mittelwerte der aktuellsten drei Jahrgänge (2015-2018) mit den drei ältesten Jahrgängen (2005-2007); angegeben wird die prozentuale Änderung in \%-Punkten. Da es sich um eine Vollerhebung und nicht nur um eine Stichprobe handelt, wurde auf die Berechnung von statistischen Tests verzichtet. Da für diese Analysen keine Mikrodaten zur Verfügung standen, konnte neben einer 


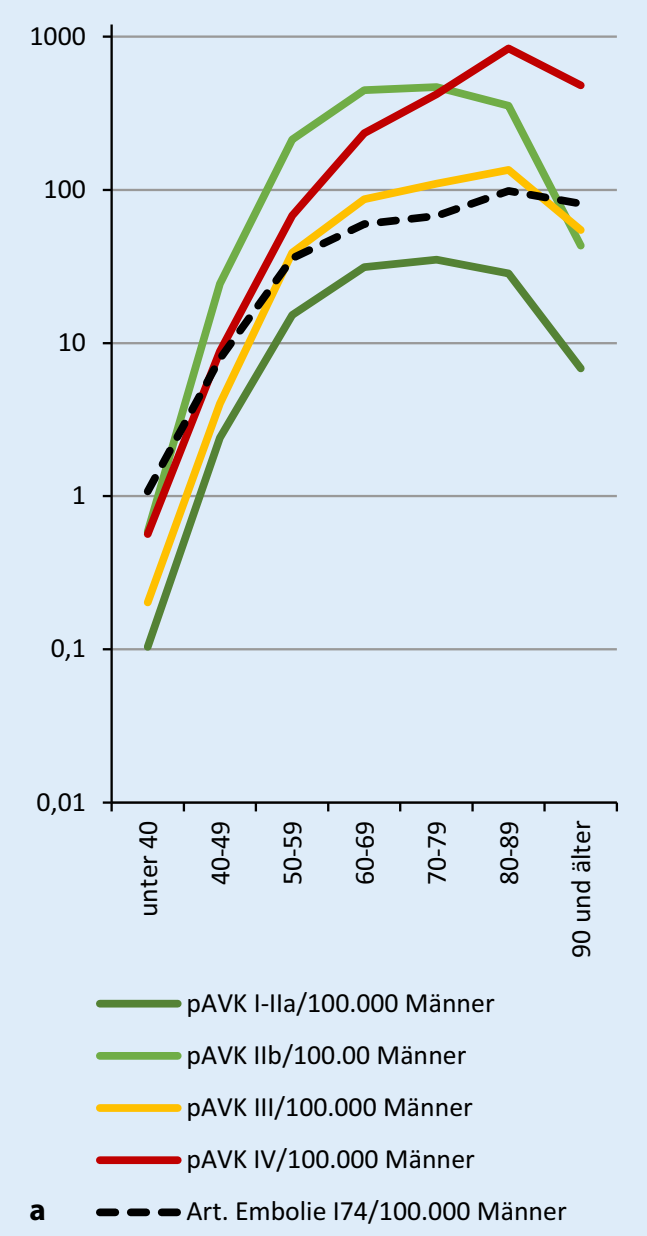

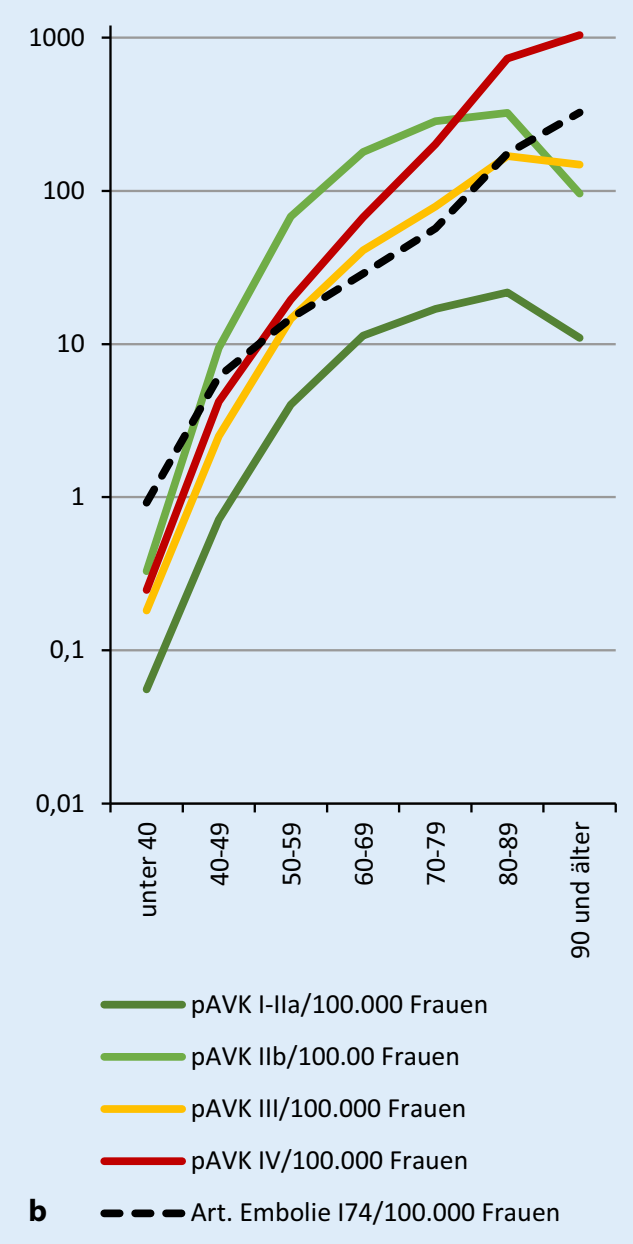

Abb. $2 \varangle$ Status quo in 2018 - Altersspezifische Krankenhausinzidenz (pAVK und arterielle Embolie/Thrombose). a Männer, b Frauen. (Falldefinition siehe Tab. 5)
Alters- und Geschlechtsstandardisierung nicht nach weiteren Variablen adjustiert werden. Ebenso konnten Interaktionen von Variablen nicht berechnet werden. Sensitivitätsanalysen wurden nicht durchgeführt.

\section{Ergebnisse}

\section{Inzidenz}

Die absolute Anzahl an Fällen mit pAVK I-IV stieg von 139.003 in 2005 auf max. 196.055 in 2016 an und lag in 2018 bei 191.491 (siehe - Abb. 1a). Die absoluten Fallzahlen der arteriellen Embolie/Thrombose stiegen zwischen 2005 und 2012 von 18.037 auf 21.742 an und fielen dann wieder auf 19.136 (in 2018) ab. Der Zeitverlauf der altersstandardisierten Werte war analog, die Krankenhausinzidenz der pAVK I-IV betrug zuletzt 231 pro 100.000 Einwohner(EW), die der arteriellen Embolie/Thrombose 23 pro $100.000 \mathrm{EW}$ (siehe - Abb. 1b). Die nach Alter und Geschlecht differenzierte Krankenhausinzidenz für 2018 ist in $\bullet$ Abb. 2 dargestellt. Während die Claudicatio intermittens (Stadium IIb) bei Patientinnen und Patienten unter 70 Jahren die höchste Inzidenz aufwies, dominiert bei Patientinnen und Patienten über 70 Jahren das Stadium IV. Die regionale Verteilung der Krankenhausinzidenz der pAVK und arteriellen Embolie/Thrombose pro $100.000 \mathrm{EW}$ ist in -Abb. 3 dargestellt. Die höchste Krankenhausinzidenz der pAVK von 340 bis 355 pro $100.000 \mathrm{EW}$ wiesen Bremen, das Saarland, Thüringen und SachsenAnhalt auf; die niedrigste Baden-Württemberg, Bayern und Rheinland-Pfalz (206 bis 212 pro $100.000 \mathrm{EW}$ ). Die arterielle Embolie/Thrombose wurde im Saarland am häufigsten diagnostiziert (34 pro $100.000 \mathrm{EW}$ ) und in Baden-
Württemberg deutlich seltener (20 pro 100.000 EW). Die Gesamtanzahl an Belegungstagen pro 100.000 Einwohner war für Männer tendenziell höher als für Frauen und stieg mit dem Alter an. Für die pAVK waren diese Werte etwa 10-mal so hoch wie für die arterielle Embolie/Thrombose (• Abb. 4).

\section{Versorgung und Therapieverfahren}

Die absolute Anzahl an offenen und endovaskulären Prozeduren sowie Prozeduren bei arterieller Embolie/Thrombose und Amputation stieg stetig von 2005 (ca. 230.000) bis 2018 (ca. 480.000) an, s. Abb. 5. Die Verteilung der versorgenden Fachabteilung ist in $\bullet$ Abb. 6 dargestellt. In Fachabteilungen/Kliniken für Gefäßchirurgie oder ungeteilten chirurgischen Abteilungen/Kliniken wurden $70,0 \%$ der Patienten mit pAVK 


\section{Originalien}

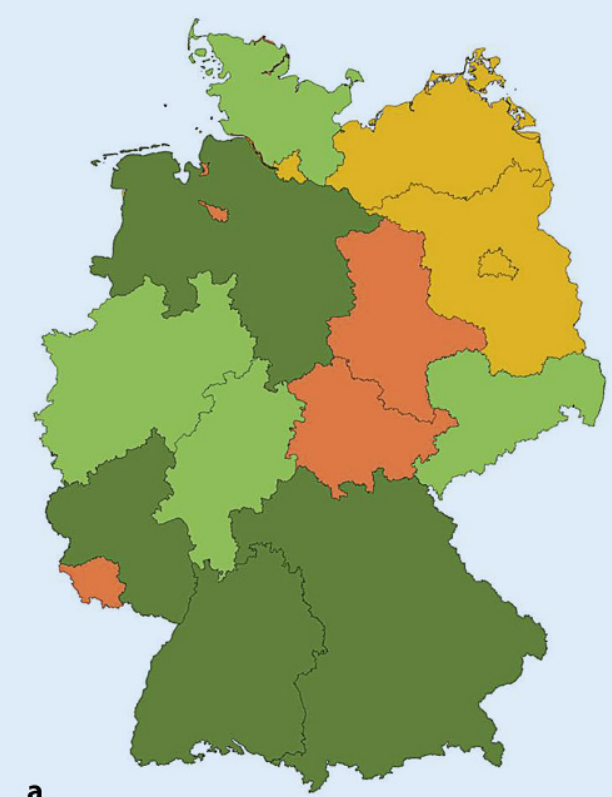

Abb. 3 A Regionale Darstellung der standardisierten KH-Inzidenz pro 100.000 Einwohner für pAVK (I70, a) und arterielle Embolie/Thrombose $(174$, b) in 2017

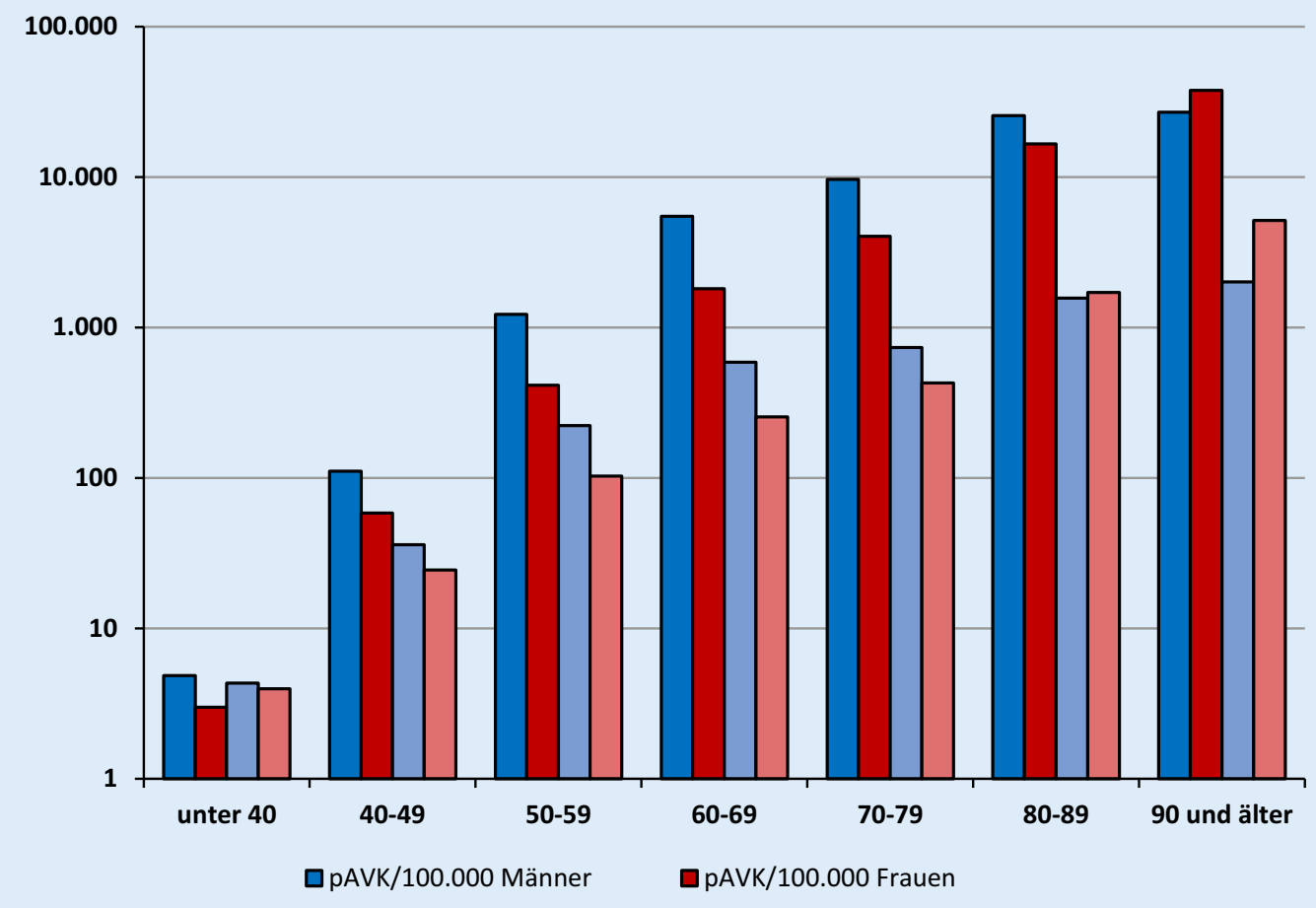

$\square$ Art. Embolie/100.000 Männer $\square$ Art. Embolie/100.000 Frauen

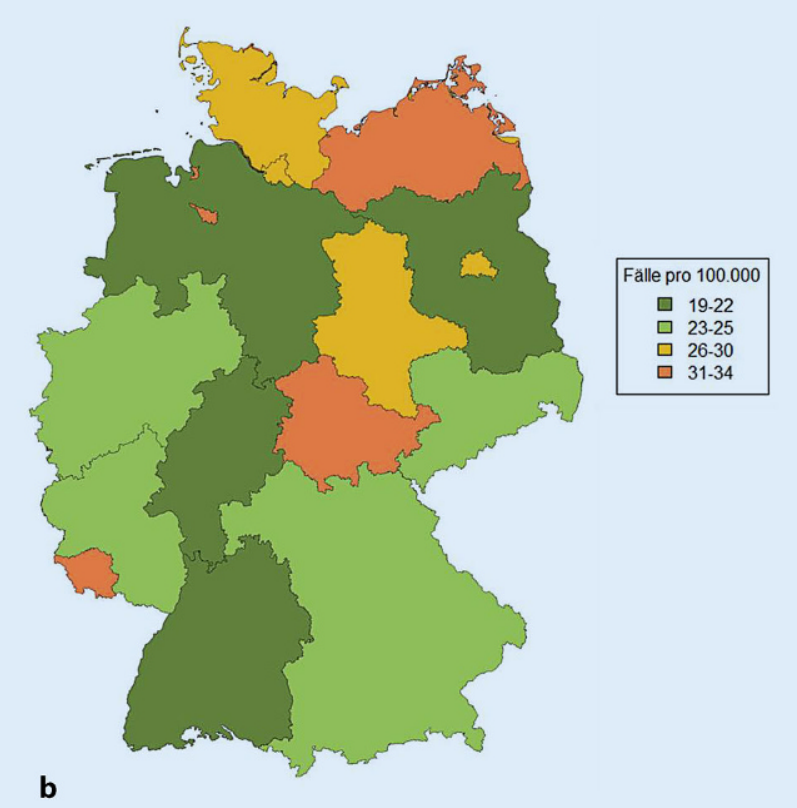

b 


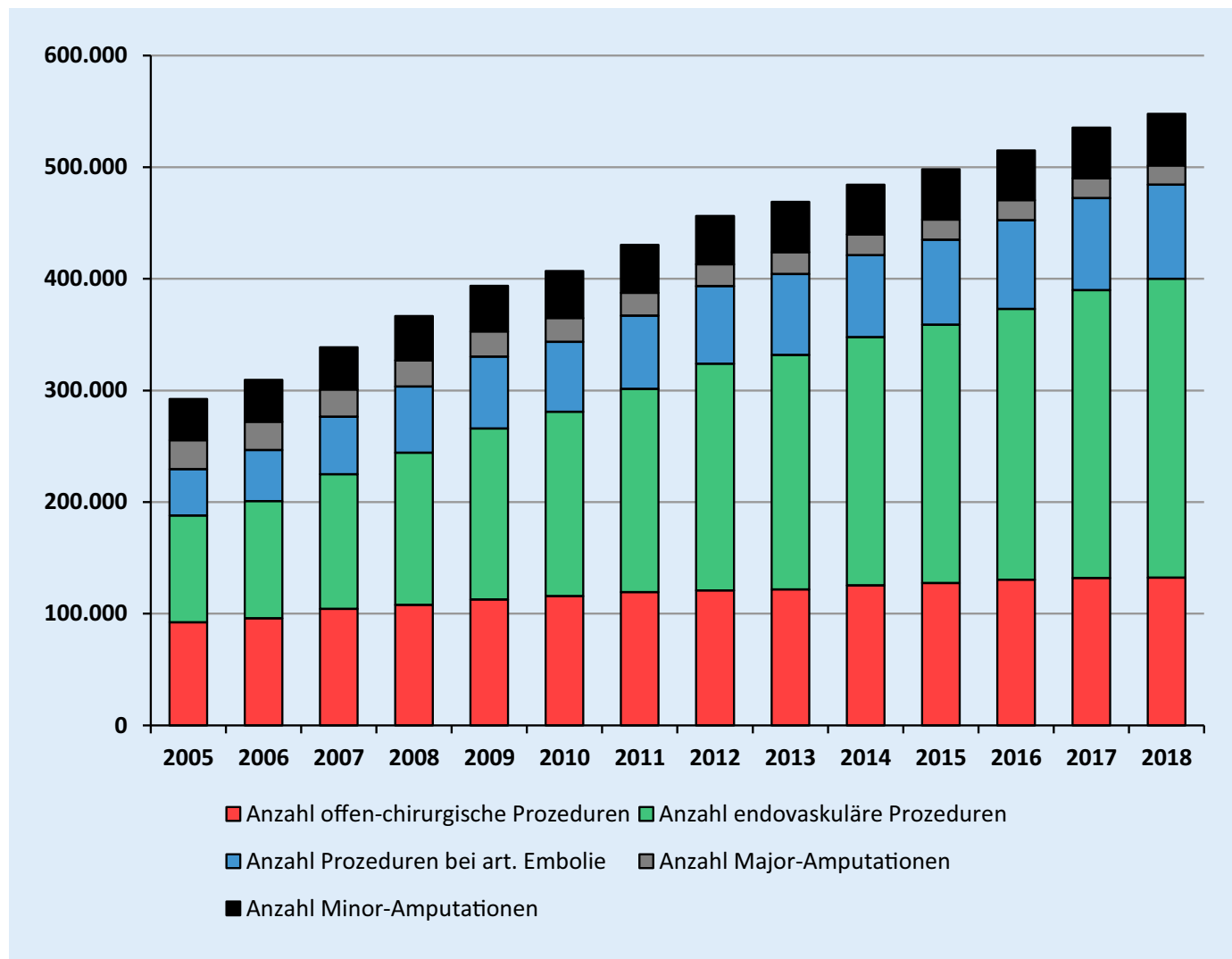

Abb. $5 \triangleleft$ Absolute Anzahl an Prozeduren von 2005 bis 2018. (Falldefinition siehe Tab. 6)

und $73,1 \%$ der Patienten mit arterieller Embolie/Thrombose behandelt.

Die absolute Anzahl aller endovaskulären Prozeduren stieg zwischen 2005 und 2018 um 139\% auf eine Gesamtzahl von 267.892 an (• Tab. 1). Den größten Zuwachs hatten die perkutane transluminale Angioplastie (PTA) der Aorta $(+157 \%)$, der künstlichen Gefäße $(+274 \%)$ und Unterschenkelgefäße $(+186 \%)$ sowie die Stentimplantation in künstliche Gefäße $(+375 \%)$ und die Gefäße des Ober- und Unterschenkels $(+267 \%$ bzw. $+300 \%)$. Mit einer Zunahme von 78.625 Prozeduren zwischen 2005 und 2018 verzeichneten PTAs am Unter- und Oberschenkel die größte absolute Zunahme.

Offen-chirurgische Eingriffe wie TEA, Bypässe und Revisionen stiegen um 35\% (•Tab. 2). Die größten Veränderungen zeigten die TEA der A. profunda femoris $(+114 \%)$, die Anastomosenrevisionen (+100\%) und Thrombendarteriektomien (TEAs) der A. iliaca externa. Absolut stiegen die TEAs der A. femoralis zwischen 2005 und 2018 am stärksten an (16.647 Prozeduren).
Offen-chirurgische Eingriffe bei arterieller Embolie/Thrombose stiegen um $56 \%$, endovaskuläre Verfahren um $141 \%$ (• Tab. 3). Den stärksten absoluten Anstieg zwischen 2005 und 2018 wiesen Inzisionen und Embolektomien am Oberschenkel (10.093) und endovaskuläre Verfahren am Oberschenkel auf (10.417).

Die Gesamtanzahl an Amputationen änderte sich kaum von 63.005 auf 63.430 (- Tab. 4). Majoramputationen nahmen um $30 \%$ ab (von 25.902 auf 17.237), Minoramputationen von 37.102 auf 46.193 um $+21 \% \mathrm{zu}$.

\section{Outcome}

Die Krankenhausmortalität der pAVK I-IV betrug in 2017 im Mittel für Männer 2,3\% und für Frauen 2,8\%, für die arterielle Embolie/Thrombose waren dies 6,8\% und 12,0\%. Die Krankenhausmortalität war klar altersabhängig und stieg bei der pAVK von $0,6 \%$ in den Altersgruppen bis 69 Jahre auf 6-7\% bei Patienten über 90 Jahre. Die nach Geschlecht differenzierte Krankenhausmortalität in den einzelnen Altersgruppen ist in Abb. 7 dargestellt.

\section{Diskussion}

\section{Krankenhausinzidenz}

In den Jahren 2005 bis 2016 zeigte sich eine stetige Zunahme der altersstandardisierten Krankenhausinzidenz, die sich zwischen 2005 und 2018 von ca. 190 auf etwas über 250 pro 100.000 Einwohner steigerte und einpendelte. Dies entspricht einer relativen Zunahme von ca. $33 \%$. Vergleichbare bundesweite Daten zur Krankenhausinzidenz sind nicht publiziert, jedoch ist die genannte relative Steigerung mit einer Analyse von Krankenkassendaten [11] und Berichten zur Prävalenz in etwa vergleichbar [4]. Die Krankenhausinzidenz zeigte sowohl für Frauen als auch für Männer und insbesondere für fortgeschrittene Krankheitsstadien einen Zusammenhang mit steigendem Alter. Diese Assoziation ist kongruent mit Daten aus anderen europäischen Staaten, die einen Anstieg der pAVK-Prävalenz von $13 \%$ in der Altersgruppe 45-54 Jahre auf ca. 30\% 


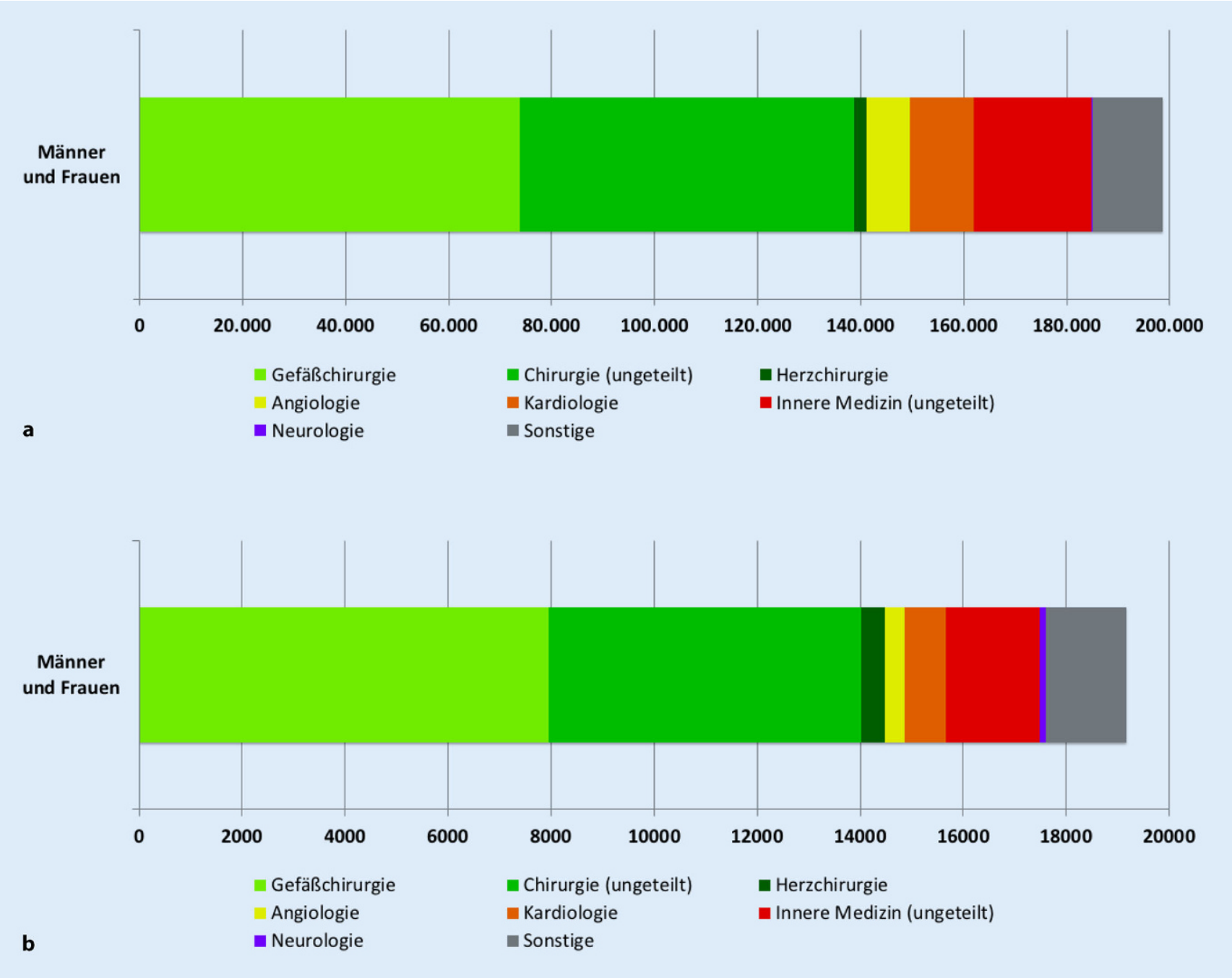

Abb. 6 \ Versorgende Fachabteilungen im Erhebungsjahr 2017. a Periphere arterielle Verschlusskrankheit Stadium I bis IV $(170,2017)$. b Arterielle Thrombose und Embolie $(174,2017)$

bei Patienten ab 65 Jahren beschrieben [12-14]. Auch in einer Analyse aus den USA konnte gezeigt werden, dass sowohl die geschlechtsspezifischen Unterschiede als auch eine Zunahme mit dem Alter bei allen Personengruppen unabhängig von ihrer ethnischen Herkunft vorhanden waren [3]. Während in der vorliegenden Studie die frühen Stadien I-IIb bei Männern und Frauen in etwa gleich häufig waren, lag die Inzidenz der pAVK III-IV bei Männern deutlich höher als bei Frauen. In einem systematischen Review zur Prävalenz der pAVK, das auf 59 internationalen Studien basierte, konnte übereinstimmend mit unseren Ergebnissen gezeigt werden, dass Männer im Vergleich zu Frauen eine höhere Prävalenz aufwei- sen und dass die Altersabhängigkeit bei Männern stärker ausgeprägt war [15]. Im Gegensatz hierzu scheint das männliche Geschlecht als Risikofaktor in Ländern mit hohem und mittlerem Einkommen im Vergleich zu Ländern mit niedrigem Einkommen gegenläufige Effekte zu zeigen [3]. Als Erklärung kommen vor allem unterschiedliche Lebens- und Ernährungsstile sowie eine vermutlich unterschiedliche Exposition mit Risikofaktoren infrage.

\section{Behandlungsverfahren}

Die Anzahl an durchgeführten Prozeduren verdoppelte sich im Beobachtungszeitraum nahezu. Diese Entwicklung wurde vor allem durch stark zuneh- mende endovaskuläre Eingriffszahlen (ca. $140 \%$ Zuwachs) und Eingriffe bei arterieller Embolie/Thrombose (ca. $+80 \%$ ) getragen, während offen-chirurgische Verfahren nur ca. um $20 \%$ zulegten. Bypässe zeigten sogar eine rückläufige Tendenz. Die Zunahme endovaskulärer Verfahren wurde u.a. auch für das abdominale Aortenaneurysma und andere Gefäßerkrankungen beobachtet und ist im Hinblick auf ein geringeres Risiko bei vergleichbarem Behandlungserfolg medizinisch plausibel zu begründen. Sowohl die Behandlung der pAVK als auch der arteriellen Thrombose und Embolie wurde überwiegend in spezialisierten Fachabteilungen für Gefäßchirurgie oder ungeteilten Abteilungen für Chirurgie durchgeführt, während Abteilungen für 


\begin{tabular}{|c|c|c|c|c|}
\hline 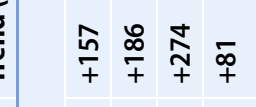 & $\stackrel{\dddot{q}}{\grave{q}} \underset{+}{+}$ & 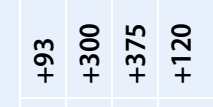 & 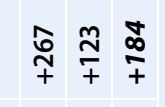 &,$\frac{\stackrel{\rho}{m}}{+}$ \\
\hline 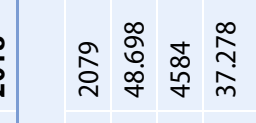 & $\begin{array}{l}\infty \\
\substack{\infty \\
\infty} \\
\infty \\
\infty\end{array}$ & 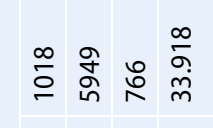 & 啽 & 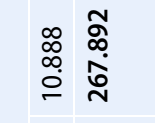 \\
\hline 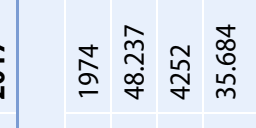 & 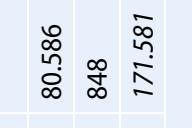 & 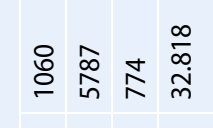 & 惫品的高 & 索总 \\
\hline 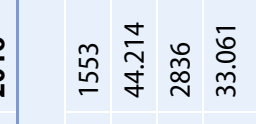 & 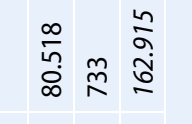 & 足 总 & 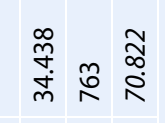 & 高 \\
\hline 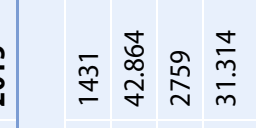 & 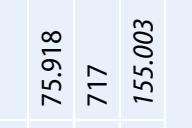 & 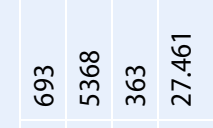 & 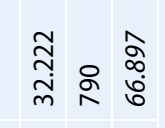 & 学誉 \\
\hline 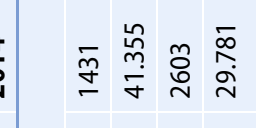 & 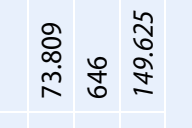 & 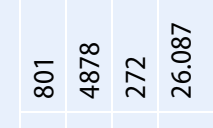 & 突 & $\stackrel{\frac{\hbar}{\pi}}{a}$ \\
\hline 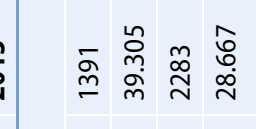 & 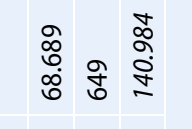 & 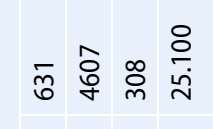 & 总 & 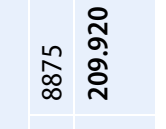 \\
\hline 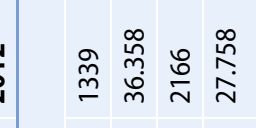 & 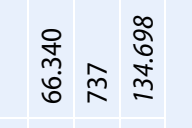 & 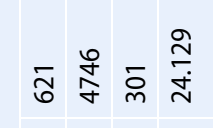 & & \\
\hline 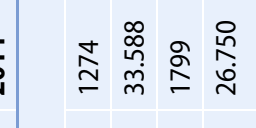 & 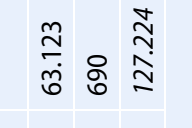 & 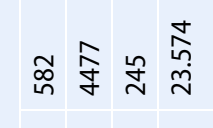 & & : \\
\hline 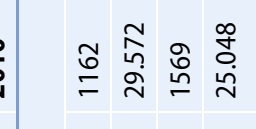 & 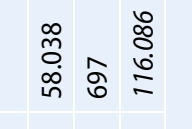 & 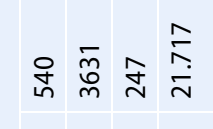 & 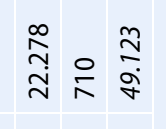 & 突 \\
\hline 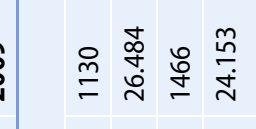 & 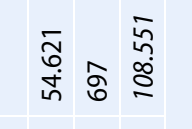 & 品 雚品 & 素云总 & 总 \\
\hline 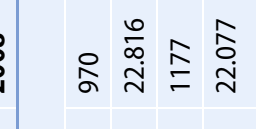 & 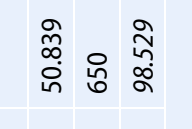 & $\approx \tilde{n}$ & 总 & 苨 \\
\hline & 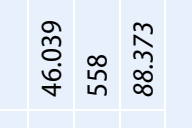 & & ذ્亏ે & \\
\hline 兽 & 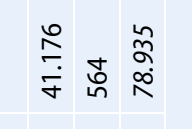 & 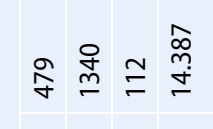 & 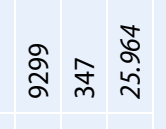 & \\
\hline & 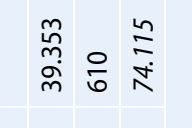 & & 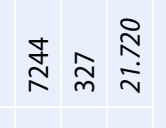 & \\
\hline 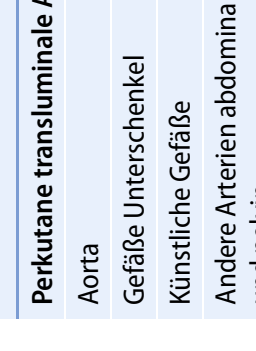 & $\begin{array}{l}\frac{\overline{\underline{J}}}{\underline{\underline{\underline{w}}}} \\
\underline{\underline{\underline{\underline{\omega}}}}\end{array}$ & 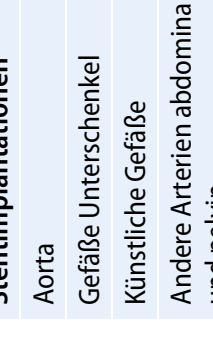 & 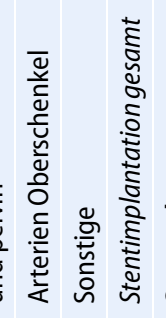 & 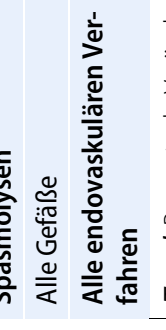 \\
\hline
\end{tabular}

Kardiologie oder Angiologie zahlenmäBig fast keine Rolle bei der stationären Versorgung spielten. Diese Ergebnisse sind vergleichbar zu früheren Publikationen $[1,7,9]$. Die Versorgungssituation im ambulanten Bereich ist auf Basis der vorliegenden Daten jedoch nicht zu beurteilen. In Zusammenschau mit der Entwicklung der Prozedurenanzahl erscheint es wahrscheinlich, dass in der traditionell „offenen Chirurgie“ zunehmend endovaskuläre Therapieverfahren etabliert und angewandt werden. Dabei muss jedoch beachtet werden, dass unterschiedliche Prozeduren (oder Revisionen), die während derselben Operation, des gleichen Aufenthalts oder im Rahmen desselben administrativen Falls beim selben Patienten durchgeführt wurden, bei der OPS bezogenen Analyse einzeln gezählt wurden. Daher ist es auch plausibel, dass auf ca. 250.000 Krankenhausfälle ca. 550.000 Prozeduren kommen.

Die Anzahl an Amputation blieb zwischen 2005 und 2018 nahezu identisch, wobei eine Verschiebung hin zu mehr Minoramputationen (+21\%) beobachtet wurde. Die abnehmenden Raten an Majoramputationen sind gleichläufig mit Beobachtungen aus den USA, Großbritannien, Italien und Finnland, jedoch gegenläufig zu Irland und Spanien [16]. Auch eine Analyse aus Deutschland, die im Gegensatz zur vorliegenden Studie Krankenkassendaten nutzte, zeigte einen vergleichbaren Trend [11]. In Bezug auf die Entwicklung von Minoramputationen zeigt sich ebenfalls ein über die Länder hinweg heterogenes Bild [16]. In einer auf Amputationen fokussierende Arbeit aus Deutschland wurden für den Zeitraum von 2005 bis 2014 nahezu identische Trends im Hinblick auf die relative Zunahme der Minorund Abnahme der Majoramputationen beschrieben [16]. Die beobachteten Unterschiede sind auf die verschiedenen Aggregationslevel der Daten, Beobachtungszeiträume und den Ausschluss bestimmter Indikationen (ICD-Gruppen $C, L, M$ und $S$ ) zurückzuführen. Die Unterschiede sind zudem dadurch erklärbar, dass die für die vorliegende Studie verwendeten Daten weder fallnoch patientenspezifisch waren. Somit 
嗢

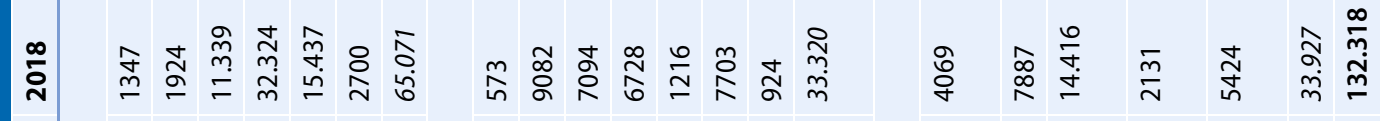

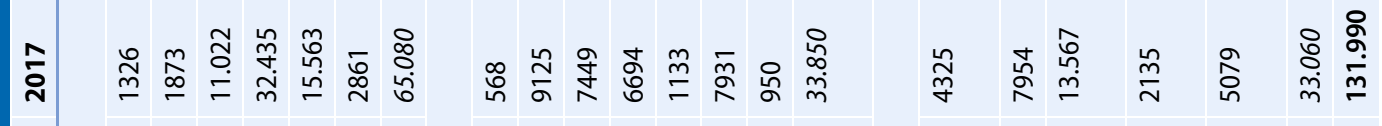

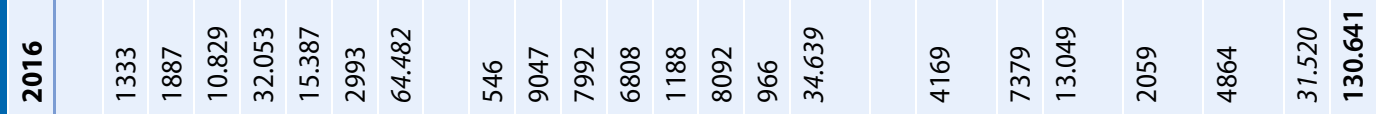

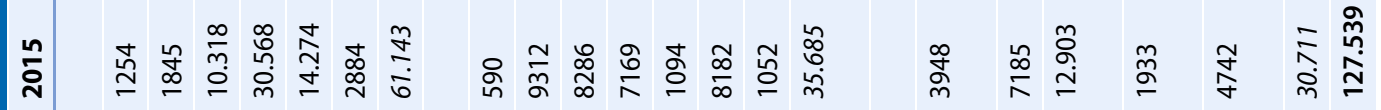

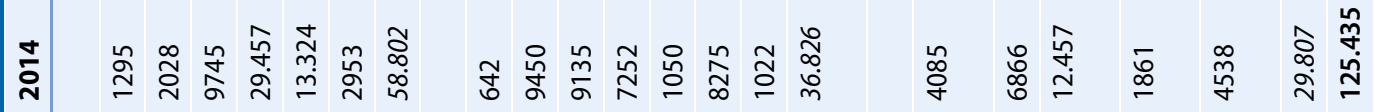

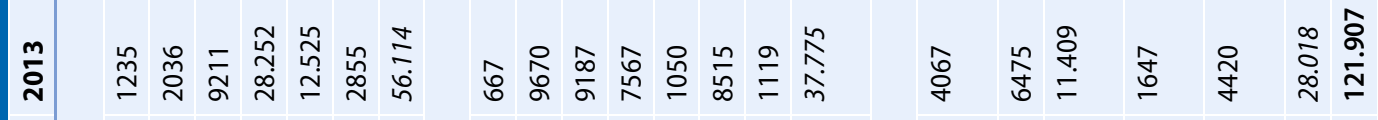
i

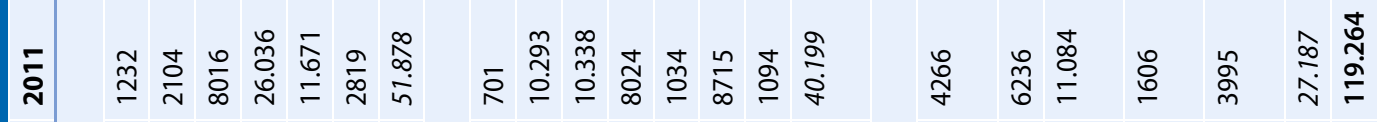

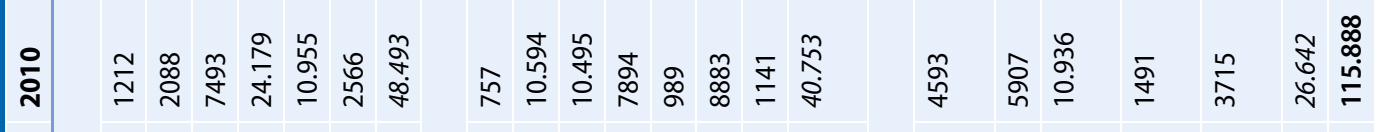

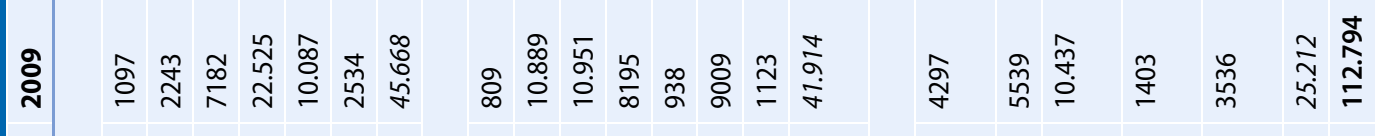

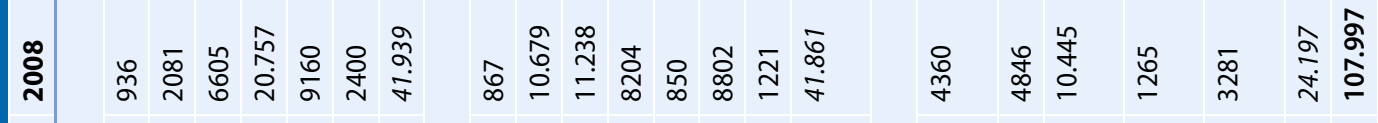

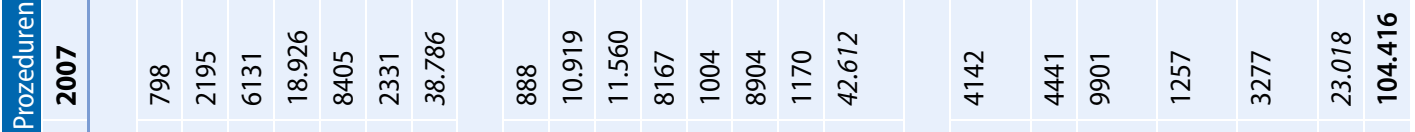
矛迹 \&

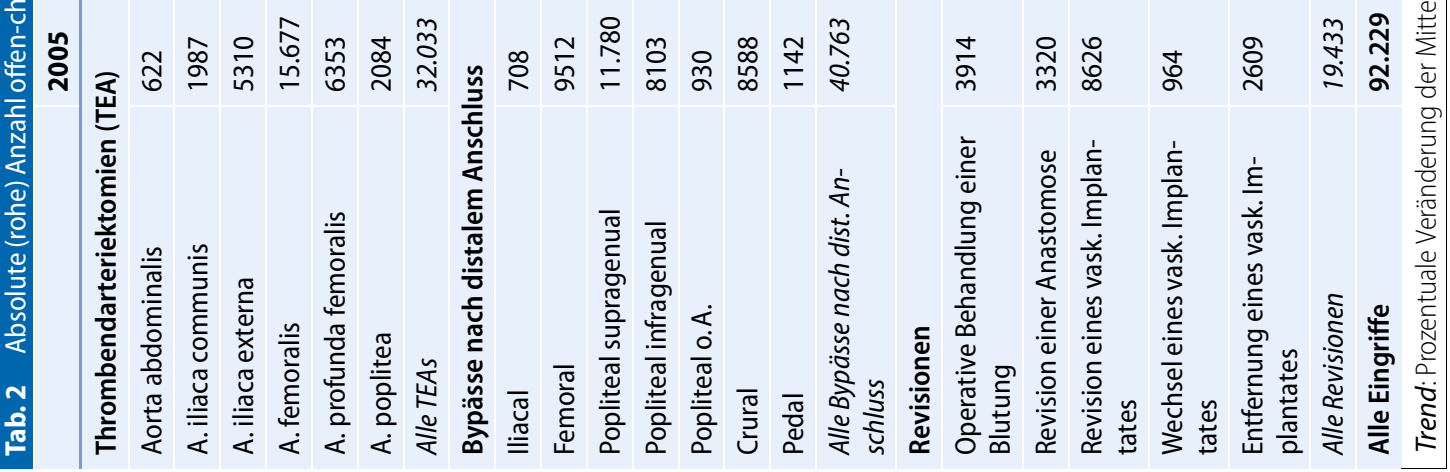




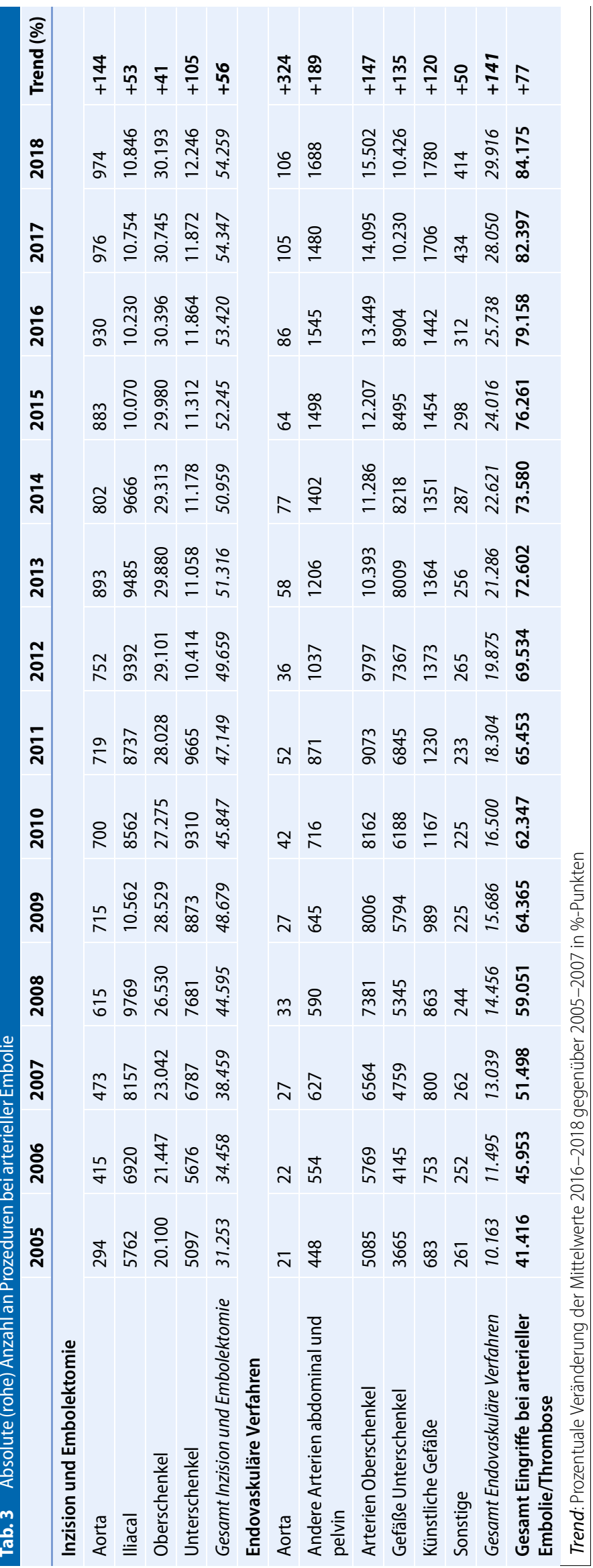

könnten die tatsächlichen Zahlen durch die im DRG-System ggf. nicht erfassten Mehrfachbehandlungen oder sequenzielle Behandlungen, die insbesondere bei umschriebenen konsekutiven Minoramputationen häufiger vorkommen, überschätzt worden sein. Auf Basis der zur Verfügung stehenden Daten konnte entweder nach ICD-10 oder nach OPS differenziert werden. Amputationen aus anderen als vaskulären Gründen, wie z.B. Tumoren oder Trauma, konnten daher nicht differenziert werden.

\section{Outcome}

Die Krankenhausmortalität war deutlich altersabhängig und stieg bei der pAVK von $0,6 \%$ in den Altersgruppen bis 69 Jahre auf 6-7\% bei Patienten über 90 Jahre. Ein Zusammenhang zwischen dem Vorhandensein einer pAVK und erhöhter Mortalität wurde bereits in mehreren Studien und Kohorten für verschiedene Endpunkte (alle, kardiovaskuläre etc.) beschrieben [3]. Allein das Vorliegen eines Ankle-Brachial-Index (ABI) von 0,9 oder weniger ist mit einer mehr als doppelt so hohen Rate an Herzinfarkten, kardiovaskulärer Mortalität oder Gesamtmortalität verbunden [8]. Ursächlich kommt hierfür weniger die pAVK an sich infrage, sondern vielmehr die koinzidenten Erkrankungen sowie eine dafür prädestinierende Risikofaktorkonstellation. Eine detaillierte Analyse des Komorbiditätsspektrums bei pAVK-Patienten wurde erst kürzlich von Kreutzburg et al. publiziert [11]. Zusammenfassend sind daher eine frühzeitige Diagnose, stadiengerechte Therapie in dafür qualifizierten und strukturell optimierten Einrichtungen sowie umfassende präventive Maßnahmen zur Verhinderung oder Verlangsamung anderer kardiovaskulärer Manifestationen unerlässlich [8].

\section{Limitationen}

Die dargestellten Zahlen und Ergebnisse basieren auf Sekundärdatenanalysen, deren grundsätzliche Biasrisiken bei Swart et al. [17] diskutiert werden. Die spezifischen methodischen Limitationen wurden bereits publiziert $[1,2,7,9]$. Die wesentlichen sind: 


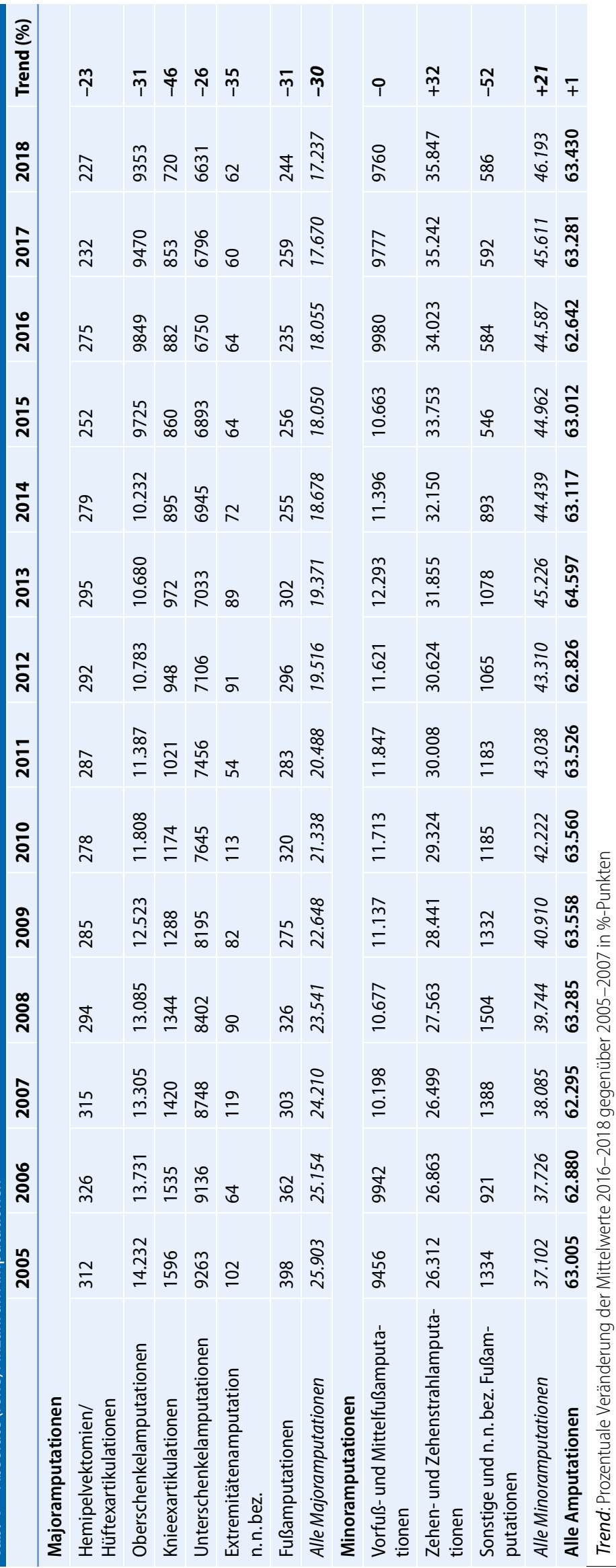

1. Bei den Daten handelt es sich um administrative Informationen und nicht um klinische Daten. Der primäre Erhebungszweck war die Krankenhausfinanzierung. Ökonomische Motive könnten, sofern sie im Rahmen von MDK-Prüfungen toleriert wurden, zu Verzerrungen geführt haben.

2. Die Daten sind fall- und nicht patientenbezogen. Mehrfache stationäre Aufenthalte können somit zu einer Überschätzung der Krankenhausinzidenz bezogen auf die individuellen Patienten führen. Aufgrund der Struktur der Datenbasis kann lediglich die Krankenhausinzidenz, nicht jedoch die populationsbezogene Inzidenz oder Prävalenz bestimmt werden. Für die Krankenhausbedarfsplanung ist jedoch neben der Einwohnerzahl, der durchschnittlichen Verweildauer und dem angestrebten Bettennutzungsgrad eben die Krankenhaushäufigkeit von Bedeutung.

3. Zur Reduktion von Verzerrungen (Bias) wurde, soweit differenzierte Daten vorlagen, eine Alters- und Geschlechtsstandardisierung durchgeführt sowie die pAVK-Stadien separat dargestellt. Aufgrund von Datenschutzvorgaben konnte dies leider nicht für alle Analysen durchgeführt werden.

4. Aufgrund des fehlenden Patientenbezugs können medizinisch zusammengehörige Behandlungen oder Komplikationen, die jedoch aus DRG-Sicht in unterschiedlichen Krankenhausaufenthalten durchgeführt wurden, nicht identifiziert werden. Ebenso können Prozeduren nicht demselben Patienten zugeordnet werden. Ob diese Eingriffe während eines Eingriffes, eines Aufenthaltes oder nach MDK-Prüfung als zusammengeführter Fall bei Wiederaufnahme abschließend kodiert wurden, kann aus den vorliegenden Daten nicht nachvollzogen werden.

5. Informationen zu Diagnostik und Therapie basieren ausschließlich auf administrativen Codes (ICD-10 und OPS). Wichtige klinische Zusatzinformationen, wie z.B. der ABI, 
Gefäßmorphologie oder Hämodynamik konnten somit nicht analysiert werden.

6. Nicht erfasst wurden Hauptdiagnosen aus T82*, da Komplikationen nicht trennscharf nur für die untere Extremität dargestellt werden können.

\section{Fazit für die Praxis}

- Die altersstandardisierte Krankenhausinzidenz der pAVK I-IV betrug zuletzt 231 pro 100.000 Einwohner (EW), die der arteriellen Embolie/ Thrombose 23 pro $100.000 \mathrm{EW}$.

- Die regionalen Unterschiede der Krankenhausinzidenz sollten zur weiteren Ursachenaufklärung sowie zur Schwerpunktsetzung im Hinblick auf Prävention und Gesundheitsförderung genutzt werden.

- Die Krankenhausinzidenz von Männern ist höher als die von Frauen; eine Altersabhängigkeit ist bei beiden Geschlechtern vorhanden.

- Die Anzahl an Prozeduren zur Behandlung einer pAVK oder arteriellen Embolie nimmt weiterhin stetig zu; insbesondere die der endovaskulären Verfahren.

- Der Großteil der stationären Versorgung von pAVK und arterieller Embolie/Thrombose erfolgt in gefäßchirurgischen oder chirurgischen Fachabteilungen oder Kliniken.

- Die Anzahl der Majoramputationen hat abgenommen, die der Minoramputationen zugenommen. Insgesamt zeigt sich keine Veränderung der Gesamtzahl von Amputationen.

- Die Krankenhausmortalität ist bei pAVK niedriger als bei arterieller Embolie/Thrombose. Beide zeigen jedoch einen klaren Zusammenhang mit einem zunehmenden Lebensalter.

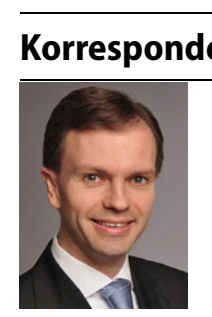

\section{MBA}

Prof. Dr. A. Kühnl, MPH

Klinik und Poliklinik für Vaskuläre und Endovaskuläre Chirurgie, Klinikum rechts der Isar, Technische Universität München

Ismaninger Straße 22, 81675 München, Deutschland a.kuehnl@tum.de

Funding. Open Access funding provided by Projekt DEAL.

\section{Einhaltung ethischer Richtlinien}

Interessenkonflikt. A. Kühnl, E. Knipfer, T. Lang, B. Bohmann, M. Trenner und H.-H. Eckstein geben an, dass kein Interessenkonflikt besteht.

Für diesen Beitrag wurden von den Autoren keine Studien an Menschen oder Tieren durchgeführt. Für die aufgeführten Studien gelten die jeweils dort angegebenen ethischen Richtlinien.

Open Access. Dieser Artikel wird unter der Creative Commons Namensnennung 4.0 International Lizenz veröffentlicht, welche die Nutzung, Vervielfältigung, Bearbeitung, Verbreitung und Wiedergabe in jeglichem Medium und Format erlaubt, sofern Sie den/die ursprünglichen Autor(en) und die Quelle ordnungsgemäßnennen, einen Link zur Creative Commons Lizenz beifügen und angeben, ob Änderungen vorgenommen wurden.

Die in diesem Artikel enthaltenen Bilder und sonstiges Drittmaterial unterliegen ebenfalls der genannten Creative Commons Lizenz, sofern sich aus der Abbildungslegende nichts anderes ergibt. Sofern das betreffende Material nicht unter der genannten Creative Commons Lizenz steht und die betreffende Handlung nicht nach gesetzlichen Vorschriften erlaubt ist, ist für die oben aufgeführten Weiterverwendungen des $\mathrm{Ma}$ terials die Einwilligung des jeweiligen Rechteinhabers einzuholen.

Weitere Details zur Lizenz entnehmen Sie bitte der Lizenzinformation auf http://creativecommons.org/ licenses/by/4.0/deed.de.

\section{Literatur}

1. Kühnl A, Söllner H, Flessenkämper I, Eckstein H-H (2013) Status quo der Gefäßchirurgie in Deutschland.Gefässchirurgie 18(5):355-364

2. Eckstein H-H (2012) Epidemiologie und Versorgung von Gefäßpatienten in Deutschland. In: Debus ES, Gross-Fengels W (Hrsg) Operative und Interventionelle Gefäßmedizin. Springer, Berlin, Heidelberg, New York

3. Criqui MH, Aboyans V (2015) Epidemiology of peripheral artery disease. Circ Res 116(9):1509-1526

4. Fowkes FG, Aboyans V, Fowkes FJ, McDermott MM, Sampson UK, Criqui MH (2017) Peripheral artery disease: epidemiology and global perspectives. Nat Rev Cardiol 14(3):156-170

5. Allison MA, Criqui MH, McClelland RL, Scott JM, McDermott MM, Liu K et al (2006) The effect of novel cardiovascular risk factors on the ethnicspecific odds for peripheral arterial disease in the Multi-Ethnic Study of Atherosclerosis (MESA). J Am Coll Cardiol 48(6):1190-1197

6. Reinecke $H$, Unrath $M$, Freisinger $E$, Bunzemeier $H$, Meyborg M, Luders F et al (2015) Peripheral arterial disease and critical limb ischaemia: still poor outcomes and lack of guideline adherence. Eur Heart J36(15):932-938

7. Kühnl A, Söllner H, Eckstein H-H (2015) Epidemiologie und stationäre Versorgung von vaskulären Erkrankungen in Deutschland. Gefässchirurgie 20(3):204-213

8. Aboyans V, Ricco JB, Bartelink MEL, Bjorck M, Brodmann M, Cohnert T et al (2018) 2017 ESC guidelines on the diagnosis and treatment of peripheral arterial diseases, in collaboration with the European Society for Vascular Surgery (ESVS). Eur Heart J 39(9):763-816 (Document covering atherosclerotic disease of extracranial carotid and vertebral, mesenteric, renal, upper and lower extremity arteries Endorsed by: the European Stroke Organization (ESO)The Task Force for the Diagnosis and Treatment of Peripheral Arterial Diseases of the European Society of Cardiology (ESC) and of the European Society for Vascular Surgery (ESVS))

9. Eckstein $\mathrm{H}-\mathrm{H}$, Knipfer $\mathrm{E}$, Trenner $\mathrm{M}$, Kühnl $\mathrm{A}$, Söllner H (2014) Epidemiologie und Behandlung der PAVK und der akuten Extremitätenischämie in deutschen Krankenhäusern von 2005 bis 2012. Gefässchirurgie 19(2):117-126

10. Spindler J (2019) Fallpauschalenbezogene Krankenhausstatistik: Diagnosen und Prozeduren der Krankenhauspatienten auf Basis der Daten nach § 21 Krankenhausentgeltgesetz. In: Klauber J, Geraedts M, Friedrich J, Wasem J (Hrsg) KrankenhausReport 2019. Springer, Berlin, Heidelberg

11. Kreutzburg T, Peters F, Riess HC, Hischke $S$, Marschall U, Kriston L et al (2020) Editor's choice-comorbidity patterns among patients with peripheral arterial occlusive disease in Germany: a trend analysis of health insurance claims data. Eur JVasc Endovasc Surg 59(1):59-66

12. Olinic DM, Spinu M, Olinic M, Homorodean C, Tataru DA, Liew A et al (2018) Epidemiology of peripheral artery disease in Europe: VAS Educational Paper. Int Angiol 37(4):327-334

13. Cacoub P, Cambou JP, Kownator S, Belliard JP, Beregi JP, Branchereau A et al (2009) Prevalence of peripheral arterial disease in high-risk patients using ankle-brachial index in general practice: a cross-sectional study. Int J Clin Pract 63(1):63-70

14. Cimminiello C, Kownator S, Wautrecht JC, Carvounis CP, Kranendonk SE, Kindler B et al (2011) The PANDORA study: peripheral arterial disease in patients with non-high cardiovascular risk. Intern Emerg Med 6(6):509-519

15. Song P, Rudan D, Zhu Y, Fowkes FJ, Rahimi K, Fowkes FGR et al (2019) Global, regional, and national prevalence and risk factors for peripheral artery disease in 2015: an updated systematic review and analysis. Lancet Glob Health 7(8):e1020-e30

16. Kroger K, Berg C, Santosa F, Malyar N, Reinecke H (2017) Lower limb amputation in Germany. Dtsch Arztebl Int 114(7):130-136

17. Swart E, Ihle P, Gothe H, Matusiewicz D (2014) Routinedaten im Gesundheitswesen, 2. Aufl. Hogrefe, Bern 